\title{
The Construction and Analysis of the Aberrant lncRNA-miRNA- mRNA Network in Adipose Tissue from Type 2 Diabetes Individuals with Obesity
}

\author{
Wei Hu $\mathbb{D}^{\mathbb{D}},{ }^{1}$ Yuanlin Ding $\mathbb{D}^{1},{ }^{1}$ Shu Wang $\mathbb{D}^{1},{ }^{1}$ Lin $X u \mathbb{D}^{1},{ }^{1}$ and Haibing $Y u \mathbb{D}^{1,2}$ \\ ${ }^{1}$ Department of Epidemiology and Medical Statistics, School of Public Health, Guangdong Medical University, Dongguan, \\ Guangdong, China \\ ${ }^{2}$ Key Laboratory of Prevention and Management of Chronic Kidney Disease of Zhanjiang City, Affiliated Hospital of Guangdong \\ Medical University, Zhanjiang, Guangdong, China
}

Correspondence should be addressed to Haibing Yu; hby616688@gdmu.edu.cn

Received 31 October 2019; Revised 12 February 2020; Accepted 12 March 2020; Published 8 April 2020

Academic Editor: Daniela Foti

Copyright (C) 2020 Wei Hu et al. This is an open access article distributed under the Creative Commons Attribution License, which permits unrestricted use, distribution, and reproduction in any medium, provided the original work is properly cited.

Background. The prevalence of obesity and type 2 diabetes mellitus (T2DM) has become the most serious global public health issue. In recent years, there has been increasing attention to the role of long noncoding RNAs (lncRNAs) in the occurrence and development of obesity and T2DM. The aim of this work was to find new lncRNAs as potential predictive biomarkers or therapeutic targets for obesity and T2DM. Methods. In this study, we identified significant differentially expressed mRNAs (DEmRNAs) and differentially expressed lncRNAs (DElncRNAs) between adipose tissue of individuals with obesity and T2DM and normal adipose tissue (absolute $\log _{2} \mathrm{FC} \geq 1$ and $\mathrm{FDR}<0.05$ ). Then, the lncRNA-miRNA interactions predicted by miRcode were further screened with a threshold of MIC $>0.2$. Simultaneously, the mRNA-miRNA interactions were explored by miRWalk 2.0. Finally, a ceRNA network consisting of lncRNAs, miRNAs, and mRNAs was established by integrating lncRNA-miRNA interactions and mRNA-miRNA interactions. Results. Upon comparing adipose tissue from individuals with obesity and T2DM and normal adipose tissues, 364 significant DEmRNAs, including 140 upregulated and 224 downregulated mRNAs, were identified in GSE104674; in addition, 231 significant DEmRNAs, including 146 upregulated and 85 downregulated mRNAs, were identified in GSE133099. GO and KEGG analyses have shown that downregulated DEmRNAs in GSE104674 and GSE133099 were associated with obesity- and T2DM-related biological pathways, such as lipid metabolism, AMPK signaling, and insulin resistance. Furthermore, 28 significant DElncRNAs, including 14 upregulated and 14 downregulated lncRNAs, were found. Based on the predicted lncRNA-miRNA and mRNA-miRNA relationships, we constructed a competitive endogenous RNA (ceRNA) network, including five lncRNAs, ten miRNAs, and 15 mRNAs. KEGG-GSEA analysis revealed that four lncRNAs (FLG-AS1, SNAI3-AS1, AC008147.0, and LINC02015) in the ceRNA network were related to the biological pathways of metabolic diseases. Conclusions. Through ceRNA network analysis, our study identified four new lncRNAs that may be used as potential biomarkers and therapeutic targets of obesity and T2DM, thus laying a foundation for future clinical studies.

\section{Introduction}

Obesity is a complex multifactorial disease caused by an imbalance between energy intake and consumption. In addition, obesity is a common complication of type 2 diabetes mellitus (T2DM) patients and is significantly associated with an increased risk of T2DM [1-3]. Studies have shown that approximately $80 \%-90 \%$ of T2DM patients can be attributed to overweight or obese [4]. Obese men are seven times more likely to develop T2DM than individuals with a healthy weight range, and obese women have a 12 -fold higher risk [5]. In the United States, the prevalence of obesity is as high as $39.6 \%$ ( $41.1 \%$ for women and $37.9 \%$ for men) [6]. At present, the trend of obesity seems to be stable in most developed countries, but in developing countries, the prevalence of obesity is increasing towards the current level in the United 
TABLE 1: Basic information of the datasets from GEO.

\begin{tabular}{lcccccc}
\hline Dataset ID & Platform & First author & Year & Region & Sample size (T2DM/N) & Types \\
\hline GSE133099 & GPL16791 & Rajan MR & 2019 & Sweden & $6 / 6$ & lncRNA, miRNA, mRNA \\
GSE104674 & GPL16558 & Stenvers DJ & 2019 & Netherlands & $24 / 24$ & mRNA \\
\hline
\end{tabular}

T2DM: type 2 diabetes mellitus; $\mathrm{N}$ : normal.

States [7]. The pathophysiological regulatory network of obesity and T2DM has always been a hot spot for scientists and a difficult point of research. An increasing number of studies have suggested that long noncoding RNA (lncRNA) plays an important role in the occurrence and development of obesity and T2DM $[8,9]$.

lncRNAs are transcripts longer than 200 bases with low protein-coding potential that were originally thought to be by-products of RNA polymerase II transcription and considered "noise" in gene transcription [8, 10-12]. Despite their lack of protein coding capacity, there is increasing evidence that lncRNAs are widely involved in the regulation of gene expression. lncRNAs are generally considered to be important regulators of adipogenesis and adipocyte metabolism [13-17]. IncRNAs also play a role in the pathogenesis of T2DM and T2DM-related complications [18-24]. Competitive endogenous RNA (ceRNA) was first proposed by Salmena et al. [25] and was defined as a class of noncoding RNAs that bind to miRNA. In recent years, an increasing number of reports have showed that lncRNAs can act as ceRNAs by competitively binding to microRNAs (miRNAs), inhibiting miRNA activity and regulating mRNA expression. The lncRNA Gm15290 sponges miR-27b to promote PPAR $\gamma$ mediated adipogenesis in vitro and to increase fat deposition and body weight in high-fat diet- (HFD-) fed mice [26]. The lncRNA H19 acts as a ceRNA of miR-30a to enhance the expression of the downstream C8orf4, modulating adipogenic differentiation in human adipose tissuederived mesenchymal stem cells [27]. The lncRNA NEAT1miR-181b-mLST8 is involved in regulating the mTOR signaling pathway in a T2DM-related ceRNA network [28]. The regulation of the IncRNA H19-miR-140-HE4 axis has a certain effect on hyperglycemia [29]. The lncRNA MEG3 promotes ATF4 expression by competitively inhibiting miR-214, leading to hepatic insulin resistance [30].

The theory of ceRNA has been widely used in the pathogenesis of diseases such as cancer, muscular dystrophy, and neurodegenerative diseases. However, studies on ceRNAs in metabolic diseases, especially obesity and T2DM, are limited. In this study, we identified differentially expressed mRNAs and lncRNAs between adipose tissue of individuals with obesity and T2DM and normal adipose tissues. Then, the IncRNA-miRNA interactions predicted by miRcode were further screened according to $\mathrm{MIC}>0.2$. Simultaneously, the mRNA-miRNA interactions were explored by miRWalk 2.0. Finally, a ceRNA network consisting of lncRNAs, miRNAs, and mRNAs was established by integrating lncRNA-miRNA interactions and mRNAmiRNA interactions. The aim of this work was to find new lncRNAs as potential predictive biomarkers or therapeutic targets for obesity and T2DM.

\section{Materials and Methods}

2.1. Collection of RNA-seq Datasets. The Gene Expression Omnibus (GEO, https://www.ncbi.nlm.nih.gov/geo) database was searched to identify all datasets that have evaluated mRNA, miRNA, and lncRNA expression in adipose tissue samples of T2DM patients with obesity [31]. The following medical subject headings (MeSH) were used for the search: ("diabetes mellitus" OR "type 2, diabetes mellitus" OR “T2DM") AND “obesity" AND ("adipose tissue” OR "fatty tissue"). The search was restricted to human samples of adipose tissue with a minimum of 3 healthy controls (no T2DM or obesity) and 3 obese T2DM patients. Finally, two RNA sequencing (RNA-seq) datasets, GSE133099 and GSE104674, were obtained. The GSE104674 dataset contains 24 patient samples and 24 healthy control samples based on the GPL16558 AB 5500 Genetic Analyzer (Homo sapiens) platform. The GSE133099 dataset contains 6 patient samples and 6 health control samples based on the GPL16791 Illumina HiSeq 2500 (Homo sapiens) platform (dataset-related information is listed in Table 1).

2.2. Identification of Differentially Expressed $m R N A s$ and lncRNAs. The ensemble ID of samples was converted by annoE 1.0.3 (https://github.com/ChrisLou-bioinfo/ AnnoENSG2GENE) based on GENCODE 31 (19.06.19) version [32]. The lncRNA, miRNA, and mRNA ensemble IDs that were not included in the GENCODE database were excluded. The edgeR, an $\mathrm{R}$ package for differential expression analysis of RNA-seq expression profiles with biological replication, was used to identify significant differentially expressed mRNAs (DEmRNAs) and differentially expressed lncRNAs (DElncRNAs) in samples from patients with T2DM and obesity and in normal samples [33]. All $q$ values used the false discovery rate (FDR) to correct the statistical significance for multiple testing. DEmRNAs and DElncRNAs with absolute $\log _{2} \mathrm{FC} \geq 1$ and FDR $<0.05$ were considered significant and were visualized through volcano graphs.

2.3. Gene Ontology and Kyoto Encyclopedia of Genes and Genomes Analysis of DEmRNAs. clusterProfiler, an R package for comparing biological themes among gene clusters [34], was used for Gene Ontology (GO) and Kyoto Encyclopedia of Genes and Genomes (KEGG) analysis of the significant DEmRNAs. GO was used to describe gene functions in three categories: biological process (BP), cellular component (CC), and molecular function (MF). The GO and KEGG analyses were searched for results at the significance level set at adjusted $P<0.05$.

2.4. IncRNA-miRNA-mRNA Network. The miRcode (http:// www.mircode.org/) was used to predict interactions between 


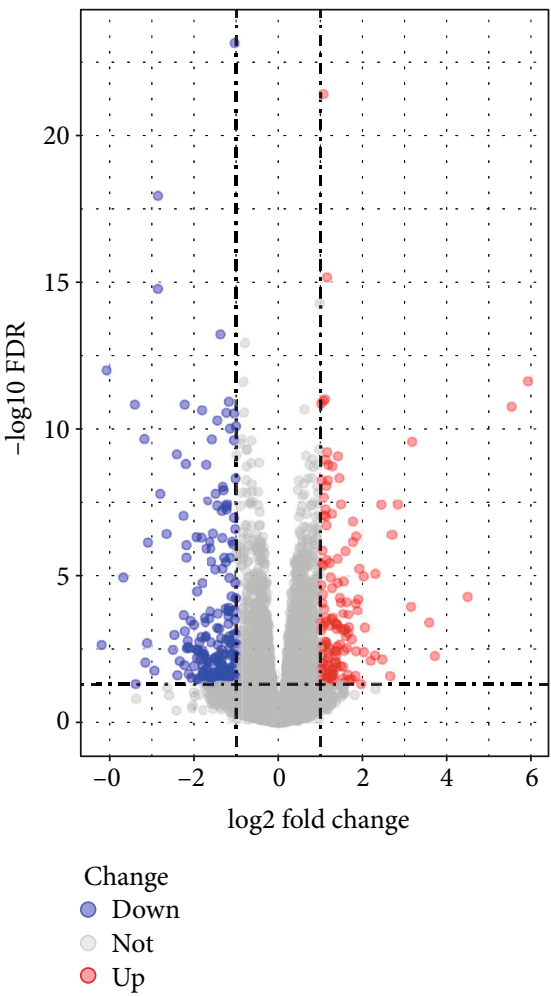

(a)

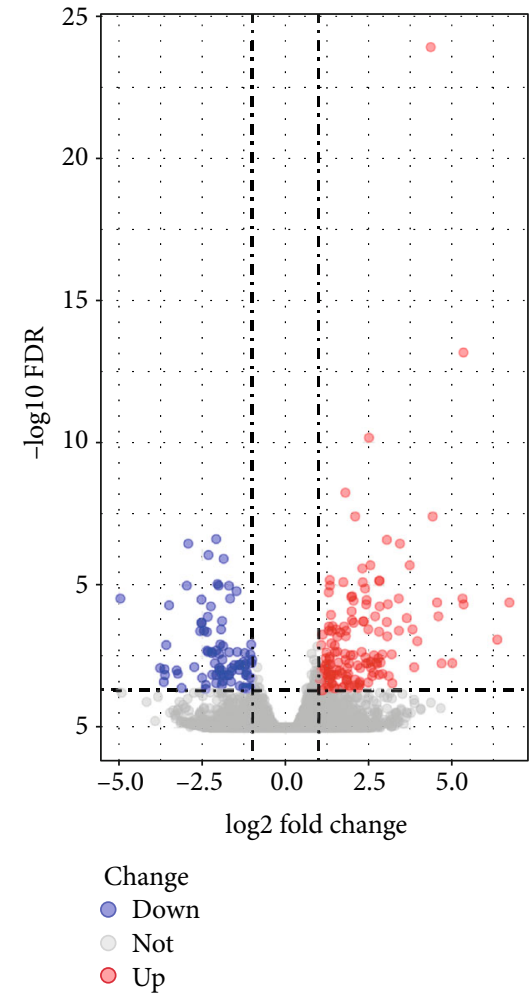

(b)

FIGURE 1: The volcano plot of significant DEmRNAs in GSE104674 (a) and GSE133099 (b). Red dots represent upregulated mRNAs, and blue dots represent downregulated mRNAs.

lncRNAs and miRNAs [35]. Moreover, the association between each probe for the DElncRNA and each available miRNA was assessed by using the maximal information coefficient (MIC) computed by minerva, an $\mathrm{R}$ package $[36,37]$. The lncRNA-miRNA interactions were screened for the significance level set at MIC > 0.2 [38].

The mRNA-miRNA interactions were predicted by miRWalk 2.0 (http://www.umm.uni-heidelberg.de/apps/ $\mathrm{zmf} / \mathrm{mirwalk} /$ ), which incorporates 12 algorithms for prediction (TargetScan, RNAhybrid, RNA22, PITA, PicTar2, miRWalk, Microt4, miRNAMap, miRDB, mirBridge, miRanda, and miRMap) [39]. The target miRNAs identified by at least seven algorithms were selected for further analysis.

Then, a lncRNA-miRNA-mRNA network was established by integrating lncRNA-miRNA interactions and mRNA-miRNA interactions and was visualized with Cytoscape 3.7.1 software [40].

2.5. KEGG Analysis for the Target miRNAs. The mirPath v.3 (http://www.microrna.gr/miRPathv3/) was used for KEGG analysis of the target miRNAs [41]. The KEGG analysis results were searched for pathways at the significance level of adjusted $P<0.05$.

2.6. Gene Set Enrichment Analysis. clusterProfiler was used for Gene Set Enrichment Analysis (GSEA) of the lncRNAs in the IncRNA-miRNA-mRNA network [42]. The Spearman correlation coefficient between lncRNAs and mRNAs in the GSE133099 dataset was calculated, and the ranked gene list was generated according to the correlation coefficient value. The KEGG-GSEA results were searched for pathways at the significance level of adjusted $P<0.05$.

\section{Results}

3.1. Identification of Differentially Expressed mRNAs. Two RNA-seq datasets (GSE133099 and GSE104674) were included in our study. A total of 364 significant DEmRNAs, including 140 upregulated and 224 downregulated mRNAs, were found in the GSE104674 dataset (Figure 1(a)); in addition, 231 significant DEmRNAs, including 146 upregulated and 85 downregulated mRNAs, were identified from the GSE133099 dataset (Figure 1(b)).

3.2. GO and KEGG Analyses of Significant DEmRNAs. The significantly upregulated and downregulated DEmRNAs of GSE133099 and GSE104674 were utilized for GO and KEGG analyses. For GO analysis of the GSE133099 dataset, when considering BPs, the top three enriched terms of the downregulated DEmRNAs were the steroid metabolic process, steroid biosynthetic process, and organic hydroxy compound biosynthetic process; the top three enriched terms of the upregulated DEmRNAs were the extracellular structure organization, extracellular matrix organization, and circulatory system processes. With regard to MF, the downregulated 


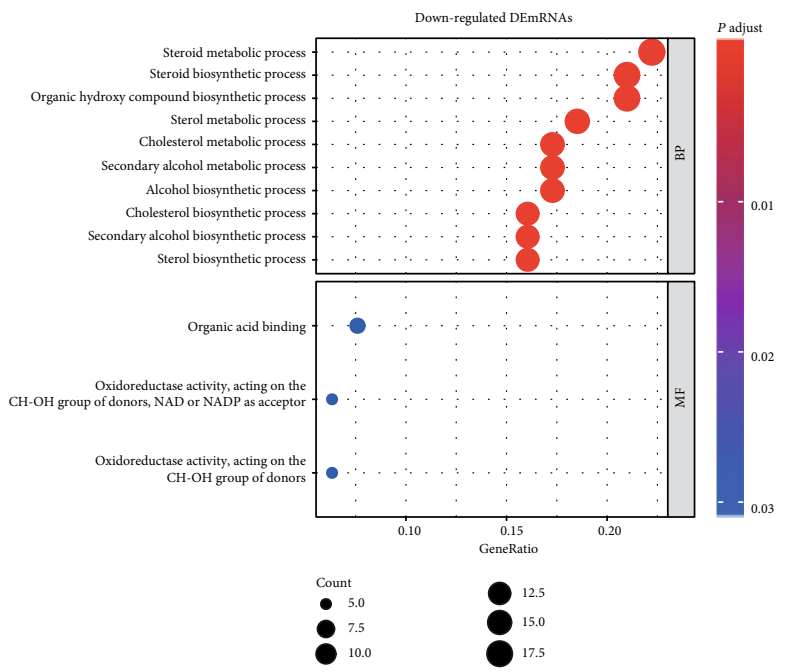

(a)

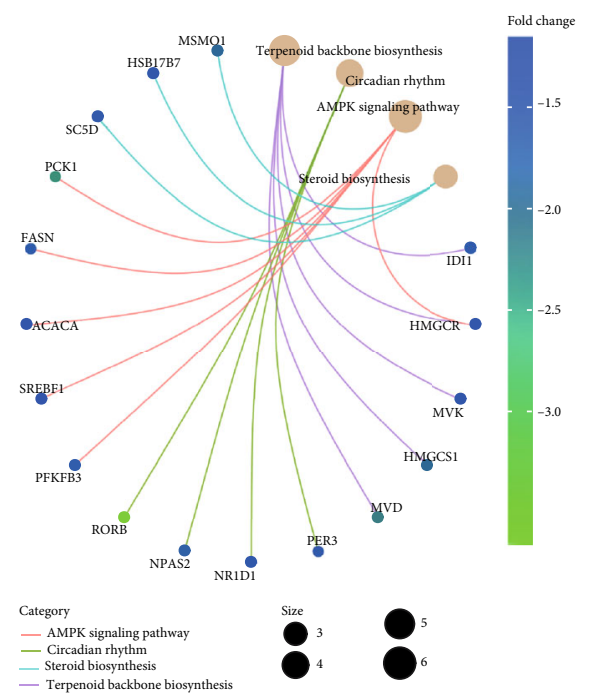

(c)

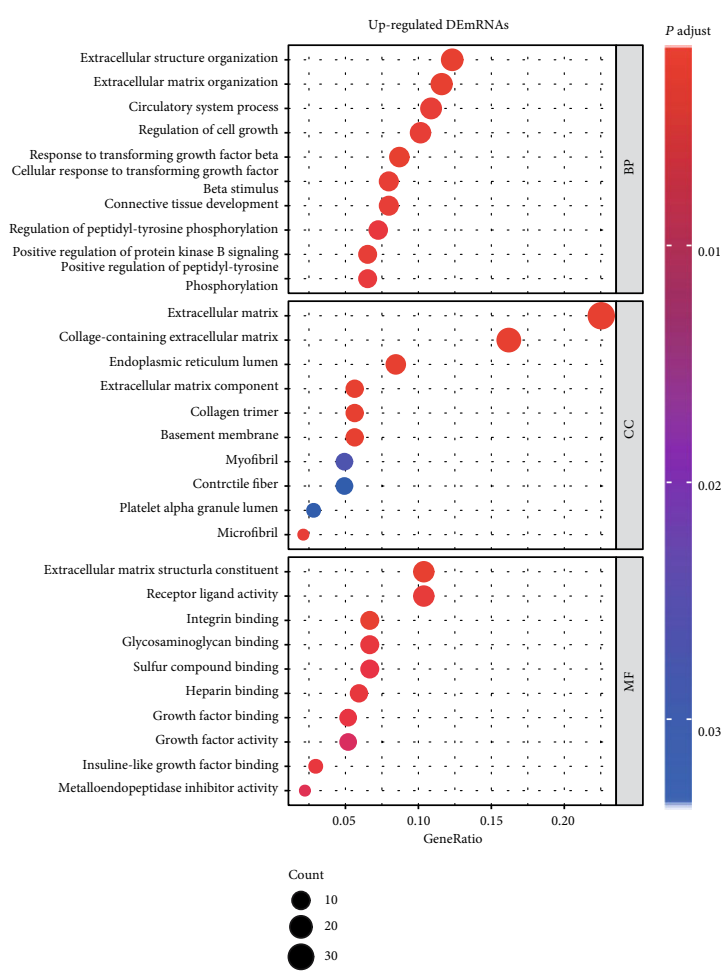

(b)

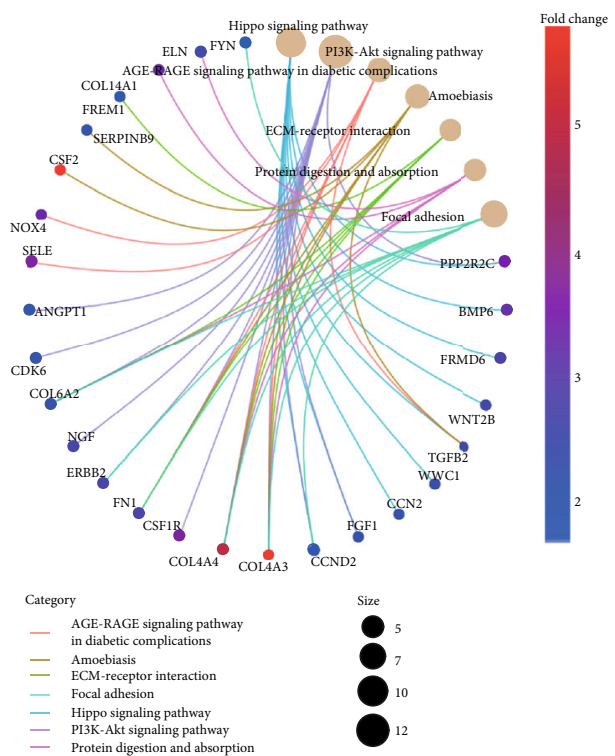

(d)

FIgURe 2: GO and KEGG analyses for significant DEmRNAs in GSE104674. The bubble charts present GO analysis results of downregulated (a) and upregulated mRNAs (b). The network charts present KEGG analysis results of downregulated (c) and upregulated mRNAs (d).

DEmRNAs were enriched in organic acid binding, oxidoreductase activity acting on the $\mathrm{CH}-\mathrm{OH}$ group of donors NAD and NADP as acceptors, and oxidoreductase activity acting on the $\mathrm{CH}-\mathrm{OH}$ group of donors; the upregulated DEmRNAs were enriched in extracellular matrix structural constituents, receptor ligand activity, and integrin binding. In terms of CCs, the extracellular matrix, collagencontaining extracellular matrix, and endoplasmic reticulum lumen are the top three enriched terms in upregulated
DEmRNAs only (Figures 2(a) and 2(b)). In the KEGG pathway enrichment analysis of the GSE133099 dataset, when considering downregulated DEmRNAs, the terpenoid backbone biosynthesis, circadian rhythm, AMPK signaling pathway, and steroid biosynthesis were enriched. With regard to upregulated DEmRNAs, the Hippo signaling pathway, PI3K-Akt signaling pathway, and AGERAGE signaling pathway in diabetic complications were the top three enriched pathways (Figures 2(c) and 2(d)). 


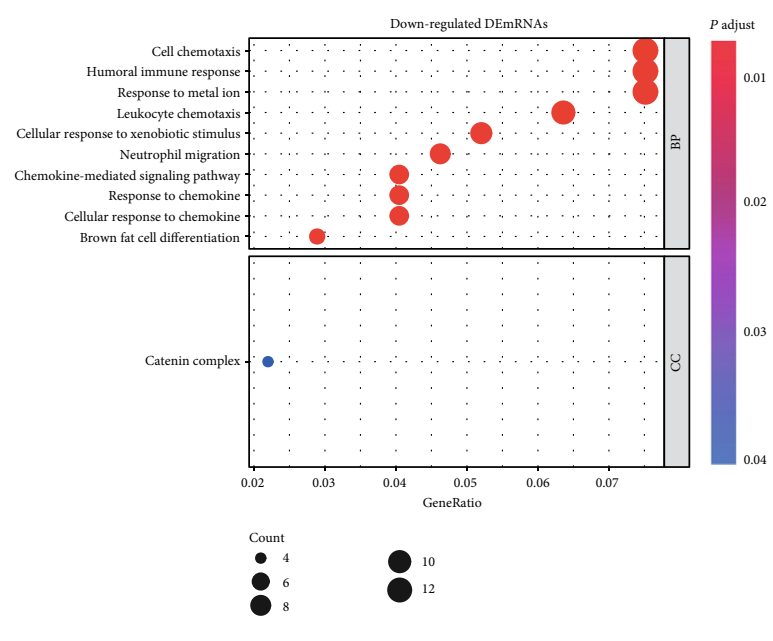

(a)

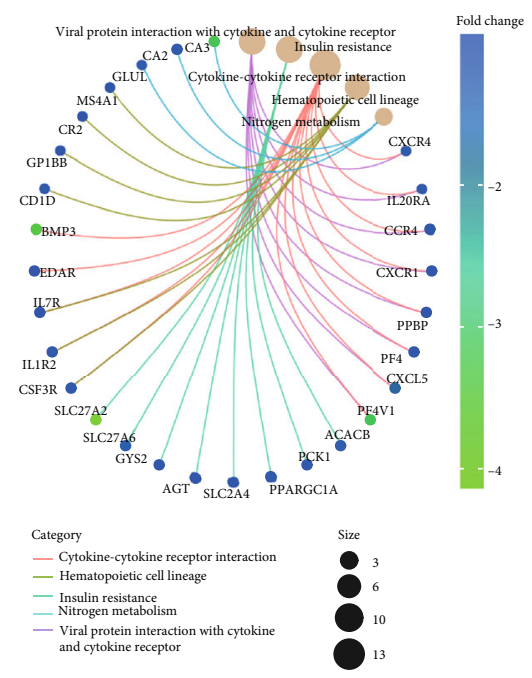

(c)
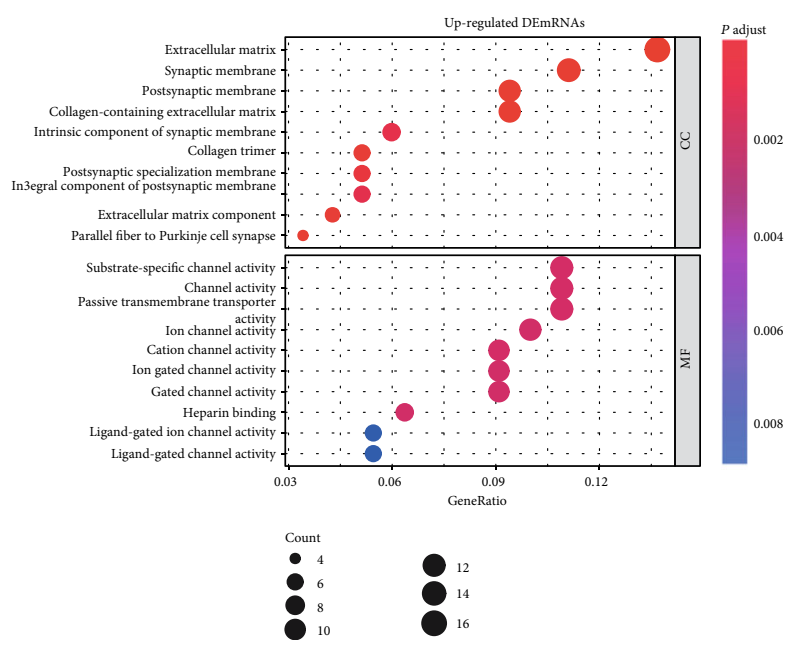

(b)

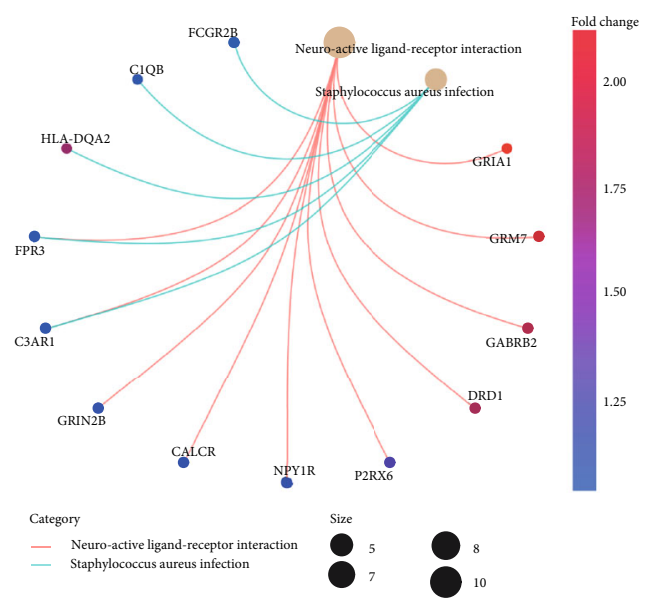

(d)

FIGURE 3: GO and KEGG analyses for significant DEmRNAs in GSE133099. The bubble charts present GO analysis results of downregulated (a) and upregulated mRNAs (b). The network charts present KEGG analysis results of downregulated (c) and upregulated mRNAs (d).

In the GO analysis of the GSE104674 dataset, when considering BPs, the top three enriched terms of the downregulated DEmRNAs were cell chemotaxis, humoral immune response, and response to metalions. With regard to MF, the upregulated DEmRNAs were enriched in substrate-specific channel activity, cation channel activity, and passive transmembrane transporter activity. In terms of CCs, the catenin complex was enriched in downregulated DEmRNAs; the extracellular matrix, synaptic membrane, and postsynaptic membrane were the top three enriched terms in upregulated DEmRNAs (Figures 3(a) and 3(b)). For KEGG pathway enrichment analysis of the GSE104674 dataset, when considering downregulated DEmRNAs, viral protein interactions with cytokine and cytokine receptors, insulin resistance, cytokine-cytokine receptor interactions, hematopoietic cell lineages, and nitrogen metabolism were enriched. With regard to upregulated DEmRNAs, neuroactive ligand-receptor interactions and Staphylococcus aureus infection were enriched (Figures 3(c) and 3(d)).
3.3. Identification of $m R N A-m i R N A$ Interactions. GO and KEGG enrichment analyses showed that significantly downregulated DEmRNAs in the GSE133099 and GSE104674 datasets were involved in obesity- and T2DM-related biological pathways, such as lipid metabolism, AMPK signaling, and insulin resistance. Therefore, the intersection of the significantly downregulated DEmRNAs in the GSE133099 and GSE104674 datasets was selected for further analysis (Figure 4(a)). Subsequently, the 15 selected mRNAs (BMP3, CA3, NDRG4, RORB, LRP1B, NTRK3, RGS2, NPC1L1, CECR2, SYT17, PCK1, SLC27A2, AZGP1, PFKFB3, and $\mathrm{ADH1B}$ ) were used to predict target miRNAs via miRWalk 2.0. A total of 1178 mRNA-miRNA pairs were found, and these pairs included 720 distinct miRNAs (Figure 4(b)).

3.4. Identification and Analysis of IncRNA-miRNA Interactions. The expression profiles of lncRNAs and miRNAs were obtained from the GSE133099 dataset. A total of 28 significant DElncRNAs, including 14 upregulated and 14 downregulated lncRNAs, were found (Figure 4(c) and Table 2). 

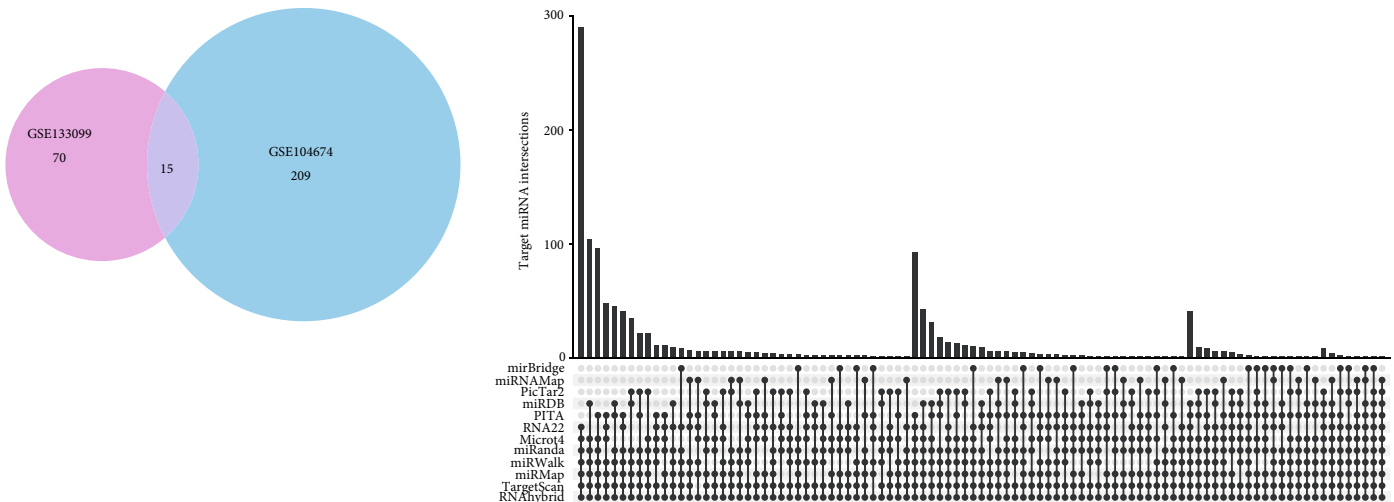

(a)

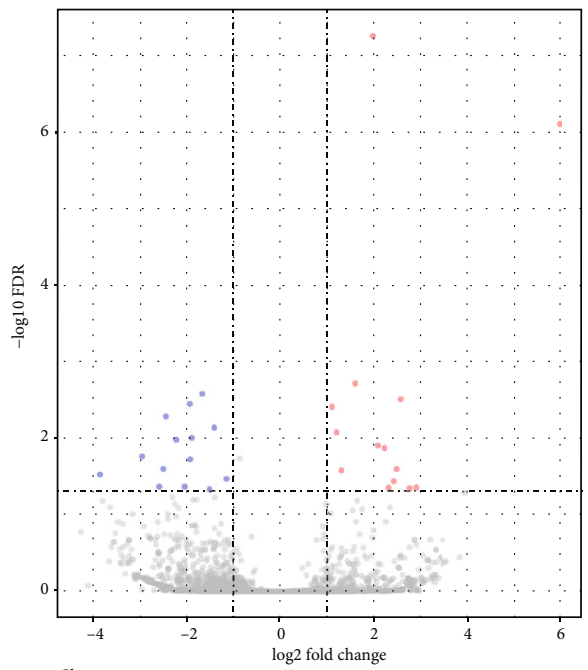

Change
$\vdots$ Down
Not
Up

(c)

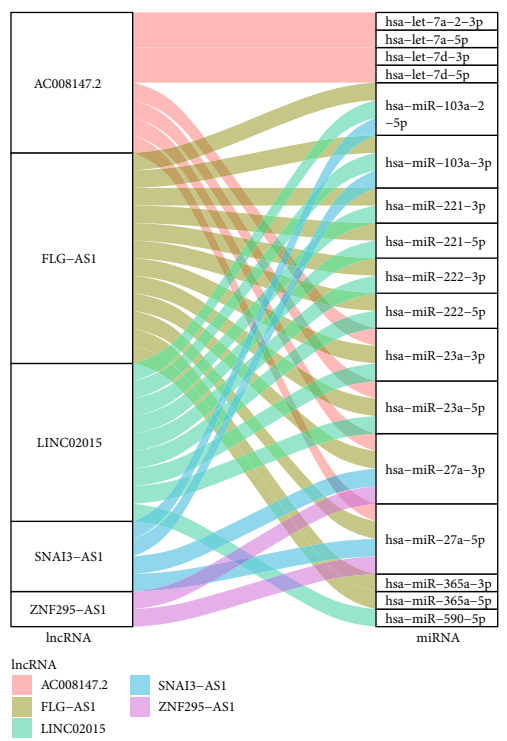

(e) (b)

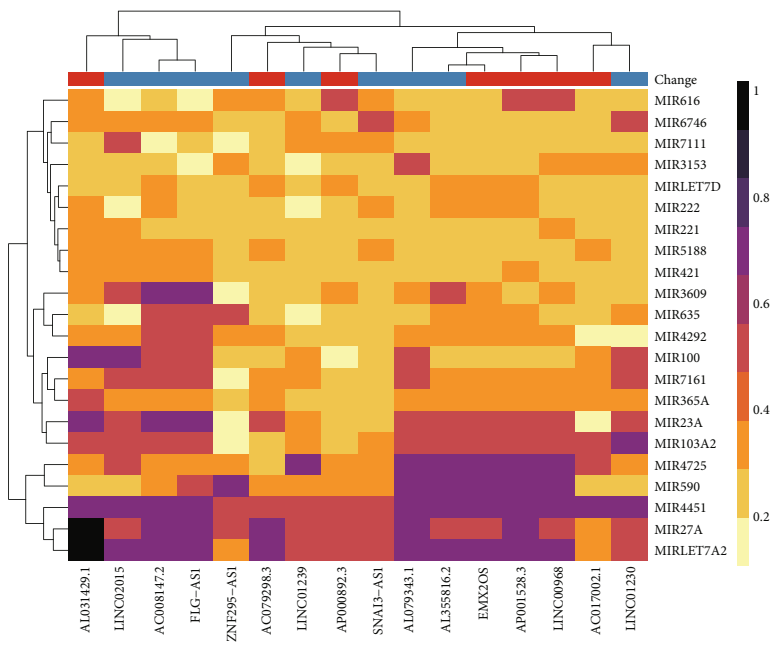

Change
Down
Up

(d)

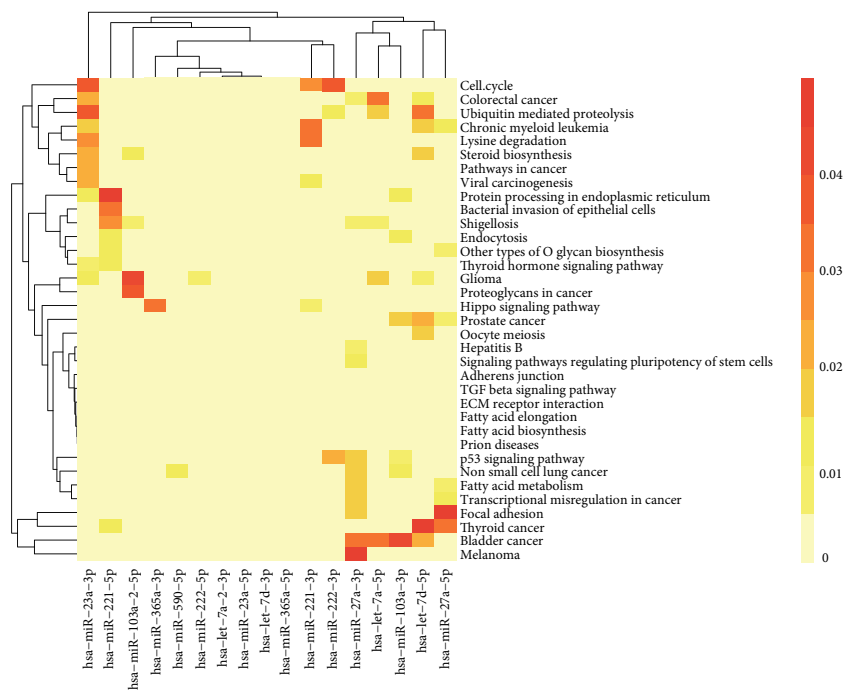

(f)

FIGURE 4: Identification of mRNA-miRNA and lncRNA-miRNA interactions. The downregulation significant DEmRNAs of GSE104674 and GSE133099 intersection was visualized by Venn plot (a). The mRNA-miRNA interactions predicted by miRWalk 2.0 were visualized by UpSet plot (b). The volcano plot of significant DElncRNAs (c). Correlation analysis between lncRNA and miRNA based on MIC (d). The lncRNA-miRNA interactions were visualized by alluvial diagram (e). KEGG pathway analysis for target miRNA (f). 
TABLE 2: The significant DElncRNAs in T2DM with obesity.

\begin{tabular}{lccccc}
\hline IncRNA & logFC & logCPM & $P$ value & FDR & Change \\
\hline AP001432.1 & -3.84 & -1.85 & $1.41 \times 10^{-4}$ & $3.05 \times 10^{-2}$ & Down \\
AC092134.1 & -2.94 & 0.69 & $6.96 \times 10^{-5}$ & $1.77 \times 10^{-2}$ & Down \\
ZNF295-AS1 & -2.58 & 1.29 & $2.32 \times 10^{-4}$ & $4.35 \times 10^{-2}$ & Down \\
AL353898.3 & -2.49 & -1.03 & $1.14 \times 10^{-4}$ & $2.58 \times 10^{-2}$ & Down \\
LINC01230 & -2.44 & 3.41 & $1.28 \times 10^{-5}$ & $5.30 \times 10^{-3}$ & Down \\
Z95114.3 & -2.22 & -0.34 & $3.49 \times 10^{-5}$ & $1.07 \times 10^{-2}$ & Down \\
SNAI3-AS1 & -2.04 & 1.25 & $2.31 \times 10^{-4}$ & $4.35 \times 10^{-2}$ & Down \\
LINC02015 & -1.93 & 5.23 & $7.79 \times 10^{-6}$ & $3.65 \times 10^{-3}$ & Down \\
AC121247.1 & -1.92 & -0.15 & $7.91 \times 10^{-5}$ & $1.92 \times 10^{-2}$ & Down \\
AL079343.1 & -1.89 & 1.57 & $3.26 \times 10^{-5}$ & $1.01 \times 10^{-2}$ & Down \\
AL355816.2 & -1.66 & 0.53 & $5.35 \times 10^{-6}$ & $2.69 \times 10^{-3}$ & Down \\
FLG-AS1 & -1.51 & 0.66 & $2.61 \times 10^{-4}$ & $4.70 \times 10^{-2}$ & Down \\
LINC01239 & -1.41 & 5.73 & $2.07 \times 10^{-5}$ & $7.46 \times 10^{-3}$ & Down \\
AC008147.2 & -1.15 & 3.43 & $1.68 \times 10^{-4}$ & $3.46 \times 10^{-2}$ & Down \\
AP001528.3 & 1.09 & 4.76 & $8.79 \times 10^{-6}$ & $3.98 \times 10^{-3}$ & Up \\
EMX2OS & 1.19 & 3.00 & $2.55 \times 10^{-5}$ & $8.61 \times 10^{-3}$ & Up \\
AC017002.1 & 1.29 & 3.16 & $1.21 \times 10^{-4}$ & $2.70 \times 10^{-2}$ & Up \\
AL031429.1 & 1.59 & 5.97 & $3.36 \times 10^{-6}$ & $1.97 \times 10^{-3}$ & Up \\
LINC00968 & 1.96 & 5.12 & $7.07 \times 10^{-12}$ & $5.63 \times 10^{-8}$ & Up \\
AL359091.4 & 2.07 & -0.36 & $4.27 \times 10^{-5}$ & $1.27 \times 10^{-2}$ & Up \\
AP000892.3 & 2.21 & 2.22 & $4.83 \times 10^{-5}$ & $1.37 \times 10^{-2}$ & Up \\
AC025580.2 & 2.30 & -0.46 & $2.47 \times 10^{-4}$ & $4.50 \times 10^{-2}$ & Up \\
LINC01173 & 2.41 & -0.04 & $1.83 \times 10^{-4}$ & $3.71 \times 10^{-2}$ & Up \\
LINC01503 & 2.46 & -0.02 & $1.15 \times 10^{-4}$ & $2.58 \times 10^{-2}$ & Up \\
AC079298.3 & 2.55 & 1.98 & $6.62 \times 10^{-6}$ & $3.18 \times 10^{-3}$ & Up \\
AC124276.2 & 2.74 & -0.24 & $2.53 \times 10^{-4}$ & $4.57 \times 10^{-2}$ & Up \\
LINC01705 & 2.89 & -0.16 & $2.44 \times 10^{-4}$ & $4.47 \times 10^{-2}$ & Up \\
AP000879.1 & 5.95 & -0.61 & $1.71 \times 10^{-10}$ & $7.94 \times 10^{-7}$ & Up \\
\hline & & & & &
\end{tabular}

Thereafter, the DElncRNA-miRNA interactions were identified by MIC correlation testing and the miRcode web tool prediction. Correlations between 16 DElncRNAs and 22 miRNAs were found after applying the MIC threshold (Figure 4(d) and Table 3). Moreover, a total of 438 DElncRNA-miRNA interactions with $18 \operatorname{lncRNAs}$ and 82 miRNAs were found by miRcode. The intersection of the above two groups was selected for further analysis; these intersections contained 35 DElncRNA-miRNA interactions with five $\operatorname{lncRNAs}$ and 17 miRNAs (Figure 4(e)).

DIANA-miRPath was exploited to explore the signaling pathways in which the 17 target miRNAs may be involved. These target miRNAs were enriched not only in cancer-related pathways but also in lipid metabolismrelated pathways, such as fatty acid biosynthesis, fatty acid metabolism, steroid biosynthesis, and fatty acid elongation (Figure 4(f)).

3.5. Construction of the IncRNA-miRNA-mRNA Network. When the IncRNA-miRNA pairs and the mRNA-miRNA pairs contained a common miRNA, they were selected for further analysis. After integrating the IncRNA-miRNA interactions and mRNA-miRNA interactions, a lncRNAmiRNA-mRNA network with five lncRNAs (FLG-AS1, SNAI3-AS1, AC008147.2, LINC02015, and ZNF295-AS1), ten miRNAs (hsa-miR-103a-3p, hsa-let-7d-5p, hsa-miR365a-3p, hsa-miR-222-3p, hsa-miR-590-5p, hsa-miR-103a2-5p, hsa-miR-23a-3p, hsa-miR-23a-5p, hsa-miR-221a-3p, and hsa-miR-27a-3p), and 15 mRNAs (BMP3, CA3, NDRG4, RORB, LRP1B, NTRK3, RGS2, NPC1L1, CECR2, SYT17, PCK1, SLC27A2, AZGP1, PFKFB3, and ADH1B) was created (Figure 5).

3.6. Gene Set Enrichment Analysis. For GSEA analysis, there were $39,51,67,85$, and zero enriched pathways for FLGAS1, SNAI3-AS1, AC008147.0, LINC02015, and ZNF295AS1, respectively (Figures 6(a)-6(d)). There were 16 intersections of enriched pathways of FLG-AS1, SNAI3-AS1, AC008147.0, and LINC02015, including cellular senescence; ubiquitin-mediated proteolysis; TGF-beta signaling; pyruvate metabolism; fatty acid degradation; peroxisomes; valine, leucine, and isoleucine degradation; steroid biosynthesis; fatty acid metabolism; fatty acid biosynthesis; glycerolipid metabolism; PPAR signaling; carbon metabolism; microRNAs in cancer; Wnt signaling; and glycolysis/gluconeogenesis (Figure 6(e)).

\section{Discussion}

With the development of the social economy, the incidence of obesity and T2DM has increased. It has been proven that the accumulation of adipose tissue, especially abdominal fat, can exacerbate insulin resistance and increase the risk of T2DM [43]. Obesity and T2DM tend to promote the occurrence and development of tumors, which imposes a huge economic burden to the world. In recent years, with the deepening of basic research on obesity and related metabolic diseases, IncRNAs have been identified to have great potential as biomarkers in fat metabolism-related diseases [12]. Recent studies have shown that lncRNAs have clinical application value and are convenient as biomarkers for disease diagnosis [44, 45]. Some lncRNAs have been reported to play a role in obesity or T2DM. However, these studies did not link obesity to T2DM. To understand more about the biological effects of lncRNAs in T2DM patients with obesity, we constructed a ceRNA network in this study, including five $\operatorname{lncRNAs}$, ten miRNAs, and 15 mRNAs.

The KEGG-GSEA results showed that four lncRNAs (FLG-AS1, SNAI3-AS1, AC008147.0, and LINC02015) in the ceRNA network were associated with cancer-related pathways, such as cellular senescence, microRNAs in cancer, and Wnt signaling. Other studies also showed that these lncRNAs were associated with cancers. As shown in the literature, FLG-AS1 may be involved in the pathogenesis of oral cancer [46]; SNAI3-AS1 can promote the growth and metastasis of hepatocellular carcinoma by inducing tumor epithelial to epithelial-mesenchymal transition [47]; LINC02015 is a protective factor for glioblastoma multiforme and is significantly upregulated in metastatic esophageal squamous cell carcinoma [48, 49]. In addition, ZNF295-AS1 is involved 
TABLE 3: Basic information of the target miRNA with MIC $>0.2$.

\begin{tabular}{|c|c|c|c|}
\hline Symbol & Genome coordinates & Stem-loop sequence & Mature sequence \\
\hline MIR616 & chr12: 57519163-57519259 & hsa-mir-616 & $\begin{array}{l}\text { hsa-miR-616-5p } \\
\text { hsa-miR-616-3p }\end{array}$ \\
\hline MIR6746 & chr11: 61878216-61878278 & hsa-mir-6746 & $\begin{array}{l}\text { hsa-miR-6746-5p } \\
\text { hsa-miR-6746-3p }\end{array}$ \\
\hline MIR7111 & chr6: $35470508-35470579$ & hsa-mir-7111 & $\begin{array}{l}\text { hsa-miR-7111-5p } \\
\text { hsa-miR-7111-3p }\end{array}$ \\
\hline MIR3153 & chr9: $89312225-89312306$ & hsa-mir-3153 & hsa-miR-3153 \\
\hline MIRLET7D & chr9: 94178834-94178920 & hsa-let-7d & $\begin{array}{l}\text { hsa-let-7d-5p } \\
\text { hsa-let-7d-3p }\end{array}$ \\
\hline MIR222 & chrX: 45747015-45747124 & hsa-mir-222 & $\begin{array}{l}\text { hsa-miR-222-5p } \\
\text { hsa-miR-222-3p }\end{array}$ \\
\hline MIR221 & chrX: 45746157-45746266 & hsa-mir-221 & $\begin{array}{l}\text { hsa-miR-221-5p } \\
\text { hsa-miR-221-3p }\end{array}$ \\
\hline MIR5188 & chr12: 124915547-124915659 & hsa-mir-5188 & hsa-miR-5188 \\
\hline MIR421 & chrX: 74218377-74218461 & hsa-mir-421 & hsa-miR-421 \\
\hline MIR3609 & chr7: $98881650-98881729$ & hsa-mir-3609 & hsa-miR-3609 \\
\hline MIR635 & chr17: 68424451-68424548 & hsa-mir-635 & hsa-miR-635 \\
\hline MIR4292 & chr9: $136830957-136831023$ & hsa-mir-4292 & hsa-miR-4292 \\
\hline MIR100 & chr11: $122152229-122152308$ & hsa-mir-100 & $\begin{array}{l}\text { hsa-miR-100-5p } \\
\text { hsa-miR-100-3p }\end{array}$ \\
\hline MIR7161 & chr6: $158609707-158609790$ & hsa-mir-7161 & $\begin{array}{l}\text { hsa-miR-7161-5p } \\
\text { hsa-miR-7161-3p }\end{array}$ \\
\hline MIR365A & chr16: 14309285-14309371 & hsa-mir-365a & $\begin{array}{l}\text { hsa-miR-365a-5p } \\
\text { hsa-miR-365a-3p }\end{array}$ \\
\hline MIR23A & chr19: 13836587-13836659 & hsa-mir-23a & $\begin{array}{l}\text { hsa-miR-23a-5p } \\
\text { hsa-miR-23a-3p }\end{array}$ \\
\hline MIR103A2 & chr20: 3917494-3917571 & hsa-mir-103a-2 & $\begin{array}{c}\text { hsa-miR-103a-2-5p } \\
\text { hsa-miR-103a-3p }\end{array}$ \\
\hline MIR4725 & chr17: 31575269-31575358 & hsa-mir-4725 & $\begin{array}{l}\text { hsa-miR-4725-5p } \\
\text { hsa-miR-4725-3p }\end{array}$ \\
\hline MIR590 & chr7: 74191198-74191294 & hsa-mir-590 & $\begin{array}{l}\text { hsa-miR-590-5p } \\
\text { hsa-miR-590-3p }\end{array}$ \\
\hline MIR4451 & chr4: $85722468-85722533$ & hsa-mir-4451 & hsa-miR-4451 \\
\hline MIR27A & chr19: $13836440-13836517$ & hsa-mir-27a & $\begin{array}{l}\text { hsa-miR-27a-5p } \\
\text { hsa-miR-27a-3p }\end{array}$ \\
\hline MIRLET7A2 & chr11: $122146522-122146593$ & hsa-let-7a-2 & $\begin{array}{c}\text { hsa-let-7a-5p } \\
\text { hsa-let-7a-2-3p }\end{array}$ \\
\hline
\end{tabular}

in the pathogenesis of epithelial ovarian cancer [50] and lung cancer [51] and can predict survival in patients with gastric cancer [52]. Though they have not been reported in metabolic diseases such as obesity and T2DM, KEGG-GSEA analysis revealed that these $\operatorname{lncRNAs}$ were related to the biological pathways of metabolic diseases, such as glucose metabolism-related pathways, lipid metabolism-related pathways, the TGF-beta signaling pathway, and the PPAR signaling pathway. Therefore, the lncRNAs in this network may play an important role in obesity and T2DM. These lncRNAs may act as ceRNAs to regulate other RNA tran- scripts by competing for shared miRNAs, thus regulating the pathogenesis of obesity and T2DM.

The miRNAs are highly conserved, single-stranded, noncoding small RNAs with a length of 18 to $25 \mathrm{nt}$ [53, 54] that can regulate gene expression by inhibiting the translation of their target mRNAs or reducing their stability at the posttranscriptional level. Studies have shown that one-third of the human genome can be regulated by miRNAs [55], which play an important regulatory role in cancers and metabolic diseases. Most miRNAs in the ceRNA network have been found to be closely related 


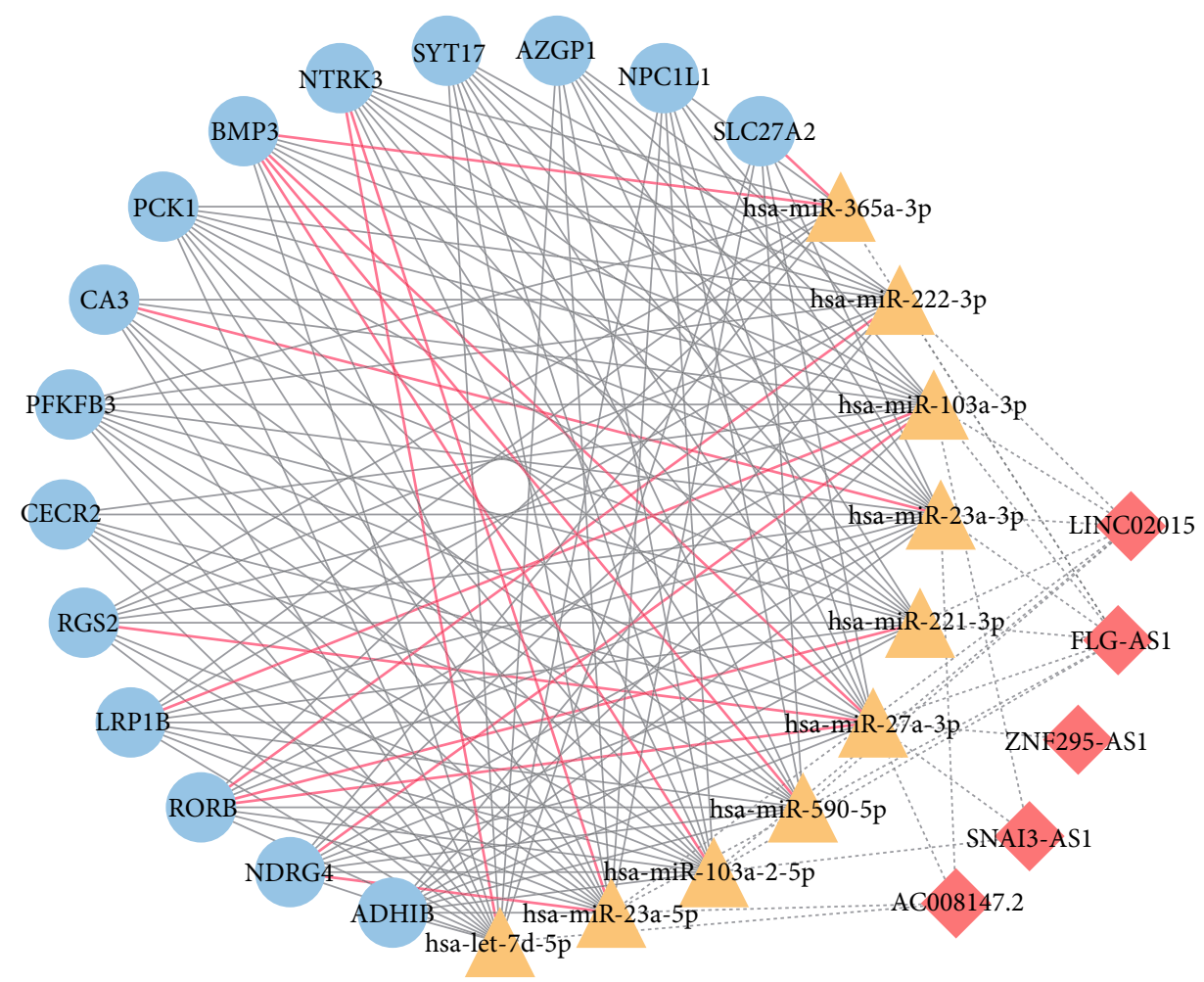

mRNAs

miRNAs

$\operatorname{lncRNAs}$

FIgURE 5: lncRNA-miRNA-mRNA network. This network consisting of five lncRNAs, ten miRNAs, and 15 mRNAs. The lncRNA-miRNA interactions are indicated by dashed lines. The mRNA-miRNA interactions are indicated by solid lines. The solid red line indicates that these interactions are confirmed by at least seven algorithms. The symbols used in the figure are indicated on the right.

to cancers [56-59]. In addition, Rohm et al. [60] found that the expression of hsa-let-7d-5p was increased in the experimental group compared with the undifferentiated control group during adipogenesis. Lozano-Bartolomé et al. [61] showed that overexpression of miR-23a-3p was involved in insulin signaling in adipocytes in vitro. miR-27a-3p plays an important role in adipogenesis [62], regulation of fat function [63], increase in glycogen storage [64], and regulation of insulin sensitivity [65]. miR-221-3p may affect insulin sensitivity and lipogenesis by regulating ANGPTL8 $[66,67]$. miR222-3p mediates the apoptosis of adipocytes in visceral fat from obese individuals and may attenuate hyperglycemia in a diabetic mouse model $[68,69]$.

Moreover, most of the mRNAs in the ceRNA network are associated with obesity and T2DM. The decreased expression of $\mathrm{ADH} 1 \mathrm{~B}$ in adipose tissue was related to obesity, systemic insulin resistance, and a decline in $\beta$ cell function [70], which may be associated with prediabetes [71]. AZGP1 may stimulate lipolysis by regulating the expression of heat-related proteins [72-75]. LRP1B gene polymorphism was associated with insulin resistance, uncontrolled emotional eating, and childhood BMI [76-78]. PCK1 has been proven to be a candidate genetic marker for the risk of diabetes and obesity
[79]. PCK1 may also participate in the progression of diabetic neuropathy [80]. PFKFB3 may be a gene that promotes "healthy obesity" [81]. The overexpression of PFKFB3 may lead to an increase in glycolysis [82]. In addition, PFKFB3/iPFK2 is involved in the anti-inflammatory and antidiabetic effects of PPAR $\gamma$ activation [83]. RGS2 may promote adipocyte differentiation and is a key regulator of pancreatic $\beta$ cell survival [84]. SLC27A2 may be a key gene in the PPAR signaling pathway, the adipocytokine signaling pathway, and the insulin resistance pathway [85]. The downregulation of SLC27A2 expression is negatively correlated with diabetes and obesity-related traits, including insulin resistance and BMI [86].

In summary, miRNAs and mRNAs in the ceRNA network were closely related to metabolic diseases such as obesity and T2DM. We have reason to believe that lncRNAs in the ceRNA network can affect the occurrence and development of obesity and T2DM by regulating the activity of target miRNAs and the expression of target mRNAs. However, there is no experimental evidence for the interaction between miRNA-target pairs in the ceRNA network. To improve reliability, these interactions should be verified experimentally. This points the way for our 

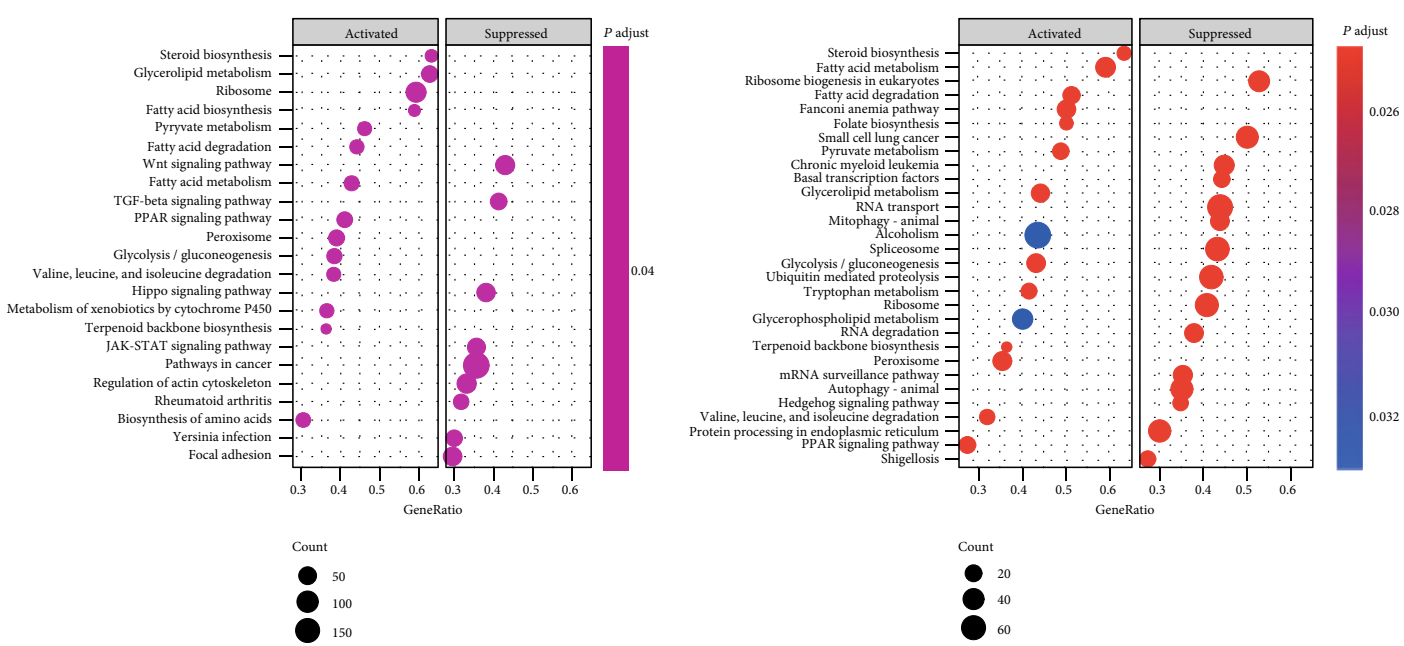

(a)

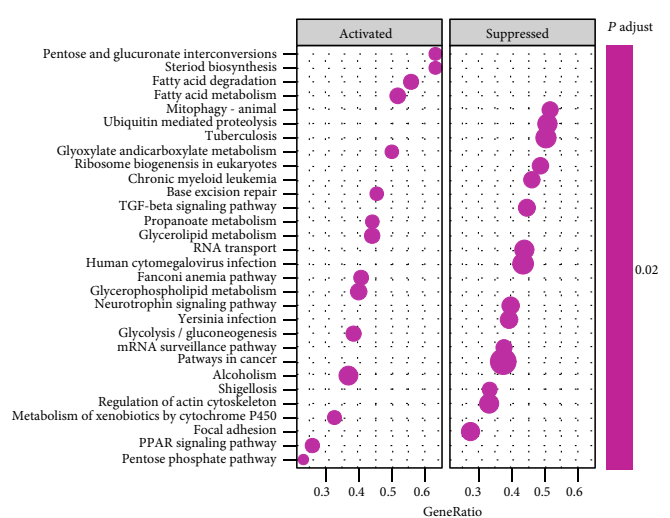

\section{:}

(c)
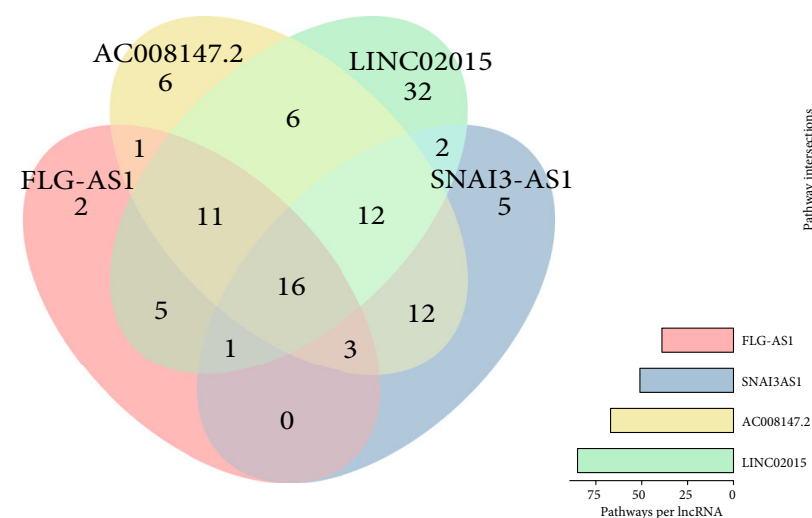

(b)

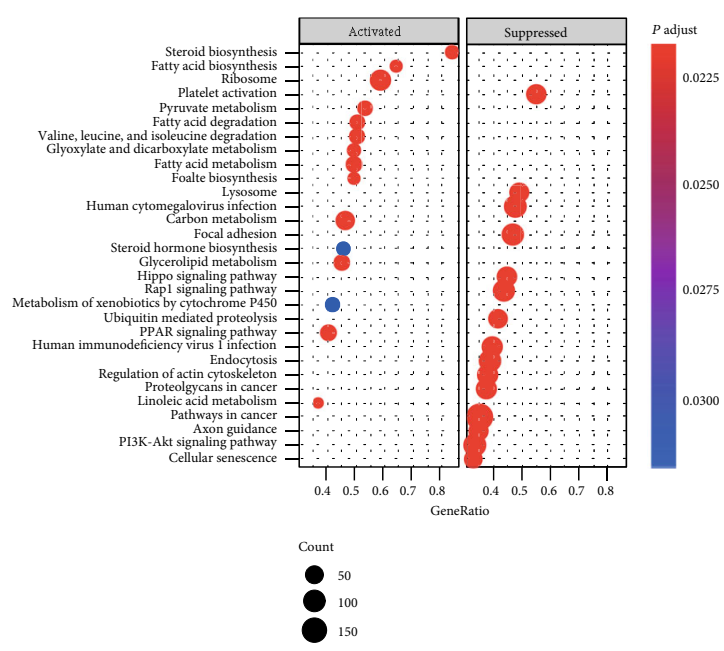

(d)

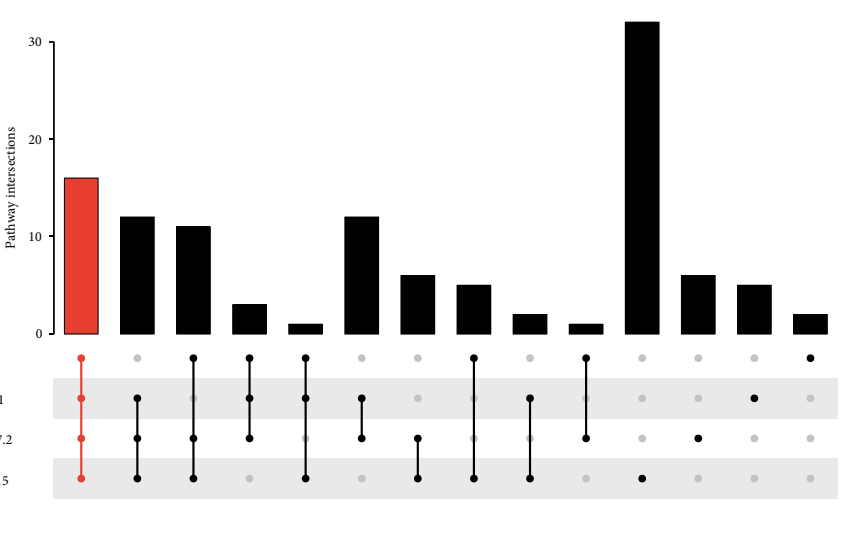

(e)

Figure 6: Gene Set Enrichment Analysis for lncRNAs in the ceRNA network. The top 30 pathways of FLG-AS1 (a), SNAI3-AS1 (b), AC008147.0 (c), and LINC02015 (d) enrichment analysis are shown by bubble charts. The Venn and UpSet plots show the pathways for the coenrichment of four lncRNAs (e).

future research. In this paper, we constructed a ceRNA network consisting of lncRNAs, miRNAs, and mRNAs, identifying four obesity- and T2DM-related lncRNAs that have not been reported in metabolic diseases. Therefore, this study may provide new targets for the pathogenesis and treatment of obesity and T2DM. 


\section{Data Availability}

The datasets of differently expressed mRNAs and lncRNAs between adipose tissue from obese T2DM individual and normal adipose tissues were acquired from GEO database, please visit: https://www.ncbi.nlm.nih.gov/geo.

\section{Conflicts of Interest}

The authors declare no conflict of interest.

\section{Authors' Contributions}

The contributions of the authors involved in this study are as follows: conceptualization: W.H. and H.Y.; methodology: W.H., S.W., L.X., and Y.D.; writing (original draft preparation): W.H. and S.W.; writing (review and editing): W.H. and S.W.; supervision: H.Y.; and funding acquisition: H.Y. and Y.D. Wei Hu and Yuanlin Ding contributed equally to this work.

\section{Acknowledgments}

The study was supported by the Medical Scientific Research Fund Project of Guangdong Province in 2018 (Grant no. B2018074), the Funds for PHD Researchers of Guangdong Medical University in 2017 (Natural Sciences, Grant no. B2017010), the Special Innovation Project of Guangdong Universities in 2019 (Natural Science, Grant no. 2019KTSCX047), the Innovation Experiment Project of Guangdong Medical University in 2019 (Grant no. ZZDG003), the Social Science and Technology Development Project of Dongguan in 2018 (General, Grant no. 20185071521339), the Young Innovative Talents Project of General Colleges and Universities in Guangdong Province (Grant no. 2018KQNCX088). We appreciate all authors for their participation and contributions. We also thank the abovementioned funds for their support.

\section{References}

[1] K. B. Smith and M. S. Smith, “Obesity statistics," Primary Care Clinics in Office Practice, vol. 43, no. 1, pp. 121-135, 2016.

[2] C. Arroyo-Johnson and K. D. Mincey, "Obesity epidemiology worldwide," Gastroenterology Clinics of North America, vol. 45, no. 4, pp. 571-579, 2016.

[3] R. A. DeFronzo, E. Ferrannini, L. Groop et al., "Type 2 diabetes mellitus," Nature Reviews Disease Primers, vol. 1, no. 1, article 15019, 2015.

[4] N. G. Docherty and C. W. le Roux, "Improvements in the metabolic milieu following Roux-en-Y gastric bypass and the arrest of diabetic kidney disease," Experimental Physiology, vol. 99, no. 9, pp. 1146-1153, 2014.

[5] J. P. Wilding, "The importance of weight management in type 2 diabetes mellitus," International Journal of Clinical Practice, vol. 68, no. 6, pp. 682-691, 2014.

[6] C. M. Hales, C. D. Fryar, M. D. Carroll, D. S. Freedman, and C. L. Ogden, "Trends in obesity and severe obesity prevalence in US youth and adults by sex and age, 2007-2008 to 20152016," JAMA, vol. 319, no. 16, pp. 1723-1725, 2018.
[7] M. Ng, T. Fleming, M. Robinson et al., "Global, regional, and national prevalence of overweight and obesity in children and adults during 1980-2013: a systematic analysis for the Global Burden of Disease Study 2013," The Lancet, vol. 384, no. 9945, pp. 766-781, 2014.

[8] Z. Chen, "Progress and prospects of long noncoding RNAs in lipid homeostasis," Molecular Metabolism, vol. 5, no. 3, pp. 164-170, 2016.

[9] C. P. Ponting, P. L. Oliver, and W. Reik, "Evolution and functions of long noncoding RNAs," Cell, vol. 136, no. 4, pp. 629641, 2009.

[10] M. Sun and W. L. Kraus, "From discovery to function: the expanding roles of long noncoding RNAs in physiology and disease," Endocrine Reviews, vol. 36, no. 1, pp. 25-64, 2015.

[11] X. Y. Zhao and J. D. Lin, "Long noncoding RNAs: a new regulatory code in metabolic control," Trends in Biochemical Sciences, vol. 40, no. 10, pp. 586-596, 2015.

[12] S. Wei, M. du, Z. Jiang, G. J. Hausman, L. Zhang, and M. V. Dodson, "Long noncoding RNAs in regulating adipogenesis: new RNAs shed lights on obesity," Cellular and Molecular Life Sciences, vol. 73, no. 10, pp. 2079-2087, 2016.

[13] A. G. Cristancho and M. A. Lazar, "Forming functional fat: a growing understanding of adipocyte differentiation," Nature Reviews Molecular Cell Biology, vol. 12, no. 11, pp. 722-734, 2011.

[14] D. R. Cooper, G. Carter, P. Li, R. Patel, J. E. Watson, and N. A. Patel, "Long non-coding RNA NEAT1 associates with SRp40 to temporally regulate PPAR $\gamma 2$ splicing during adipogenesis in 3T3-L1 cells," Genes, vol. 5, no. 4, pp. 1050-1063, 2014.

[15] J. Chen, Y. Liu, S. Lu et al., "The role and possible mechanism of lncRNA U90926 in modulating 3T3-L1 preadipocyte differentiation," International Journal of Obesity, vol. 41, no. 2, pp. 299-308, 2017.

[16] J. Sun, Y. Ruan, M. Wang et al., "Differentially expressed circulating lncRNAs and mRNA identified by microarray analysis in obese patients," Scientific Reports, vol. 6, no. 1, article 35421, 2016.

[17] R. Balakrishnan, M. A. Harris, R. Huntley, K. Van Auken, and J. M. Cherry, "A guide to best practices for Gene Ontology (GO) manual annotation," Database, vol. 2013, article bat054, 2013.

[18] S. D. Feng, J. H. Yang, C. H. Yao et al., "Potential regulatory mechanisms of lncRNA in diabetes and its complications," Biochemistry and Cell Biology, vol. 95, no. 3, pp. 361-367, 2017.

[19] Y. Kotake, T. Nakagawa, K. Kitagawa et al., "Long non-coding RNA ANRIL is required for the PRC2 recruitment to and silencing of $p 15^{\mathrm{INK} 4 \mathrm{~B}}$ tumor suppressor gene," Oncogene, vol. 30, no. 16, pp. 1956-1962, 2011.

[20] J. Fadista, P. Vikman, E. O. Laakso et al., "Global genomic and transcriptomic analysis of human pancreatic islets reveals novel genes influencing glucose metabolism," Proceedings of the National Academy of Sciences of the United States of America, vol. 111, no. 38, pp. 13924-13929, 2014.

[21] X. Zhu, Y. B. Wu, J. Zhou, and D. M. Kang, "Upregulation of lncRNA MEG3 promotes hepatic insulin resistance via increasing FoxO1 expression," Biochemical and Biophysical Research Communications, vol. 469, no. 2, pp. 319-325, 2016.

[22] G. Carter, B. Miladinovic, A. A. Patel, L. Deland, S. Mastorides, and N. A. Patel, "Circulating long noncoding RNA GAS5 
levels are correlated to prevalence of type 2 diabetes mellitus," BBA clinical, vol. 4, pp. 102-107, 2015.

[23] L. E. Abdulle, J. L. Hao, O. P. Pant et al., "MALAT1 as a diagnostic and therapeutic target in diabetes-related complications: a promising long-noncoding RNA," International Journal of Medical Sciences, vol. 16, no. 4, pp. 548-555, 2019.

[24] A. Lelli, K. A. Nolan, S. Santambrogio et al., "Induction of long noncoding RNA MALAT1 in hypoxic mice," Hypoxia, vol. 3, pp. 45-52, 2015.

[25] L. Salmena, L. Poliseno, Y. Tay, L. Kats, and P. P. Pandolfi, "A ceRNA hypothesis: the Rosetta stone of a hidden RNA language?," Cell, vol. 146, no. 3, pp. 353-358, 2011.

[26] W. Liu, C. Ma, B. Yang, C. Yin, B. Zhang, and Y. Xiao, "IncRNA Gm15290 sponges miR-27b to promote PPAR $\gamma$ induced fat deposition and contribute to body weight gain in mice," Biochemical and Biophysical Research Communications, vol. 493, no. 3, pp. 1168-1175, 2017.

[27] Z. Shao, C. Chen, W. Li, H. Ren, and W. Chen, "Assessment of the risk factors in the daily life of stroke patients based on an optimized decision tree," Technology and Health Care, vol. 27, no. S1, pp. 317-329, 2019.

[28] Z. Lin, X. Li, X. Zhan et al., "Construction of competitive endogenous RNA network reveals regulatory role of long non-coding RNAs in type 2 diabetes mellitus," Journal of Cellular and Molecular Medicine, vol. 21, no. 12, pp. 3204-3213, 2017.

[29] Y. Luo, Z. Fang, Y. Ling, and W. Luo, "LncRNA-H19 acts as a ceRNA to regulate HE4 expression by sponging miR-140 in human umbilical vein endothelial cells under hyperglycemia with or without $\alpha$-Mangostin," Biomedicine \& Pharmacotherapy, vol. 118, article 109256, 2019.

[30] X. Zhu, H. Li, Y. Wu, J. Zhou, G. Yang, and W. Wang, "IncRNA MEG3 promotes hepatic insulin resistance by serving as a competing endogenous RNA of miR-214 to regulate ATF4 expression," International Journal of Molecular Medicine, vol. 43, no. 1, pp. 345-357, 2019.

[31] R. Edgar, M. Domrachev, and A. E. Lash, "Gene expression omnibus: NCBI gene expression and hybridization array data repository," Nucleic Acids Research, vol. 30, no. 1, pp. 207$210,2002$.

[32] J. Harrow, A. Frankish, J. M. Gonzalez et al., "GENCODE: the reference human genome annotation for the ENCODE project," Genome Research, vol. 22, no. 9, pp. 1760-1774, 2012.

[33] D. J. McCarthy, Y. Chen, and G. K. Smyth, "Differential expression analysis of multifactor RNA-Seq experiments with respect to biological variation," Nucleic Acids Research, vol. 40, no. 10, pp. 4288-4297, 2012.

[34] G. Yu, L. G. Wang, Y. Han, and Q. Y. He, “clusterProfiler: an R package for comparing biological themes among gene clusters," OMICS, vol. 16, no. 5, pp. 284-287, 2012.

[35] A. Jeggari, D. S. Marks, and E. Larsson, "miRcode: a map of putative microRNA target sites in the long non-coding transcriptome," Bioinformatics, vol. 28, no. 15, pp. 2062-2063, 2012.

[36] D. N. Reshef, Y. A. Reshef, H. K. Finucane et al., "Detecting novel associations in large data sets," Science, vol. 334, no. 6062, pp. 1518-1524, 2011.

[37] D. Albanese, M. Filosi, R. Visintainer, S. Riccadonna, G. Jurman, and C. Furlanello, "Minerva and minepy: a C engine for the MINE suite and its R, Python and MATLAB wrappers," Bioinformatics, vol. 29, no. 3, pp. 407-408, 2013.
[38] K. M. Gharpure, S. Pradeep, M. Sans et al., "FABP4 as a key determinant of metastatic potential of ovarian cancer," Nature Communications, vol. 9, no. 1, article 2923, 2018.

[39] C. Sticht, C. De La Torre, A. Parveen, and N. Gretz, "miRWalk: an online resource for prediction of microRNA binding sites," PLoS One, vol. 13, no. 10, article e0206239, 2018.

[40] P. Shannon, A. Markiel, O. Ozier et al., "Cytoscape: a software environment for integrated models of biomolecular interaction networks," Genome Research, vol. 13, no. 11, pp. 24982504, 2003.

[41] I. S. Vlachos, K. Zagganas, M. D. Paraskevopoulou et al., "DIANA-miRPath v3.0: deciphering microRNA function with experimental support," Nucleic Acids Research, vol. 43, no. W1, pp. W460-W466, 2015.

[42] A. Subramanian, P. Tamayo, V. K. Mootha et al., "Gene set enrichment analysis: a knowledge-based approach for interpreting genome-wide expression profiles," Proceedings of the National Academy of Sciences of the United States of America, vol. 102, no. 43, pp. 15545-15550, 2005.

[43] J. Koska, N. Stefan, P. A. Permana et al., "Increased fat accumulation in liver may link insulin resistance with subcutaneous abdominal adipocyte enlargement, visceral adiposity, and hypoadiponectinemia in obese individuals," The American Journal of Clinical Nutrition, vol. 87, no. 2, pp. 295-302, 2008.

[44] H. Amir, M. A. Khan, S. Feroz et al., "CARLo-7-A plausible biomarker for bladder cancer," International Journal of Experimental Pathology, vol. 100, no. 1, pp. 25-31, 2019.

[45] L. Bolha, M. Ravnik-Glavač, and D. Glavač, "Long noncoding RNAs as biomarkers in cancer," Disease Markers, vol. 2017, Article ID 7243968, 14 pages, 2017.

[46] L. Feng, J. R. Houck, P. Lohavanichbutr, and C. Chen, “Transcriptome analysis reveals differentially expressed lncRNAs between oral squamous cell carcinoma and healthy oral mucosa," Oncotarget, vol. 8, no. 19, pp. 31521-31531, 2017.

[47] Y. Li, D. Guo, M. Ren et al., "Long non-coding RNA SNAI3AS1 promotes the proliferation and metastasis of hepatocellular carcinoma by regulating the UPF1/Smad7 signalling pathway," Journal of Cellular and Molecular Medicine, vol. 23, no. 9, pp. 6271-6282, 2019.

[48] W.-Z. Gao, L.-M. Guo, T.-Q. Xu, Y.-H. Yin, and F. Jia, "Identification of a multidimensional transcriptome signature for survival prediction of postoperative glioblastoma multiforme patients," Journal of Translational Medicine, vol. 16, no. 1, p. 368, 2018.

[49] F. Yang, S. Wen, Y. Zhang et al., "Identifying potential metastasis-related long non-coding RNAs, microRNAs, and message RNAs in the esophageal squamous cell carcinoma," Journal of Cellular Biochemistry, vol. 120, no. 8, pp. 1320213215, 2019.

[50] Y. Ding, D.-Z. Yang, Y.-N. Zhai et al., "Microarray expression profiling of long non-coding RNAs in epithelial ovarian cancer," Oncology Letters, vol. 14, no. 2, pp. 2523-2530, 2017.

[51] G. Xu, J. Chen, Q. Pan et al., "Long noncoding RNA expression profiles of lung adenocarcinoma ascertained by microarray analysis," PLoS One, vol. 9, no. 8, article e104044, 2014.

[52] Z. Hu, D. Yang, Y. Tang et al., "Five-long non-coding RNA risk score system for the effective prediction of gastric cancer patient survival," Oncology Letters, vol. 17, no. 5, pp. 44744486, 2019.

[53] D. P. Bartel, "MicroRNAs: genomics, biogenesis, mechanism, and function," Cell, vol. 116, no. 2, pp. 281-297, 2004. 
[54] Q. Jing, S. Huang, S. Guth et al., "Involvement of microRNA in AU-rich element-mediated mRNA instability," Cell, vol. 120, no. 5, pp. 623-634, 2005.

[55] B. P. Lewis, C. B. Burge, and D. P. Bartel, "Conserved seed pairing, often flanked by adenosines, indicates that thousands of human genes are microRNA targets," Cell, vol. 120, no. 1, pp. 15-20, 2005.

[56] R. García-Vázquez, D. Gallardo Rincón, E. Ruiz-García et al., "let-7d-3p is associated with apoptosis and response to neoadjuvant chemotherapy in ovarian cancer," Oncology Reports, vol. 39, no. 6, pp. 3086-3094, 2018.

[57] C.-L. Zhu, X. Sha, Y. Wang et al., "Circular RNA hsa_circ_ 0007142 is upregulated and targets miR-103a-2-5p in colorectal cancer," Journal of Oncology, vol. 2019, Article ID 9836819, 10 pages, 2019.

[58] M. Li, H. Guan, Y. Liu, and X. Gan, "IncRNA ZEB1-AS1 reduces liver cancer cell proliferation by targeting miR-365a3p," Experimental and Therapeutic Medicine, vol. 17, no. 5, pp. 3539-3547, 2019.

[59] Z. Q. Zheng, Z. X. Li, G. Q. Zhou et al., "Long noncoding RNA FAM225A promotes nasopharyngeal carcinoma tumorigenesis and metastasis by acting as ceRNA to sponge miR-5903p/miR-1275 and upregulate ITGB3," Cancer Research, vol. 79, no. 18, pp. 4612-4626, 2019.

[60] B. Rohm, A.-K. Holik, N. Kretschy et al., "Nonivamide enhances miRNA let-7d expression and decreases adipogenesis PPAR $\gamma$ expression in 3T3-L1 cells," Journal of Cellular Biochemistry, vol. 116, no. 6, pp. 1153-1163, 2015.

[61] J. Lozano-Bartolomé, G. Llauradó, M. Portero-Otin et al., "Altered expression of miR-181a-5p and miR-23a-3p is associated with obesity and TNF $\alpha$-induced insulin resistance," The Journal of Clinical Endocrinology and Metabolism, vol. 103, no. 4, pp. 1447-1458, 2018.

[62] C. Shi, F. Huang, X. Gu et al., "Adipogenic miRNA and metasignature miRNAs involved in human adipocyte differentiation and obesity," Oncotarget, vol. 7, no. 26, pp. 4083040845, 2016.

[63] C. Castaño, S. Kalko, A. Novials, and M. Párrizas, "Obesityassociated exosomal miRNAs modulate glucose and lipid metabolism in mice," Proceedings of the National Academy of Sciences of the United States of America, vol. 115, no. 48, pp. 12158-12163, 2018.

[64] F. Chemello, F. Grespi, A. Zulian et al., "Transcriptomic analysis of single isolated myofibers identifies miR-27a-3p and miR-142-3p as regulators of metabolism in skeletal muscle," Cell Reports, vol. 26, no. 13, pp. 3784-3797.e8, 2019.

[65] D. Dahlmans, A. Houzelle, J. A. Jörgensen et al., "Evaluation of muscle microRNA expression in relation to human peripheral insulin sensitivity: a cross-sectional study in metabolically distinct subject groups," Frontiers in Physiology, vol. 8, p. 711, 2017.

[66] N. Mononen, L.-P. Lyytikäinen, I. Seppälä et al., "Whole blood microRNA levels associate with glycemic status and correlate with target mRNAs in pathways important to type 2 diabetes," Scientific Reports, vol. 9, no. 1, article 8887, 2019.

[67] R. Mysore, F. J. Ortega, J. Latorre et al., "MicroRNA-221-3p regulates angiopoietin-like 8 (ANGPTL8) expression in adipocytes," The Journal of Clinical Endocrinology and Metabolism, vol. 102, no. 11, pp. 4001-4012, 2017.

[68] N. N. Wijayatunga, M. Pahlavani, N. S. Kalupahana et al., "An integrative transcriptomic approach to identify depot differences in genes and microRNAs in adipose tissues from high fat fed mice," Oncotarget, vol. 9, no. 10, pp. 92469261, 2018.

[69] S. Tsukita, T. Yamada, K. Takahashi et al., "MicroRNAs 106b and 222 improve hyperglycemia in a mouse model of insulin-deficient diabetes via pancreatic $\beta$-cell proliferation," eBioMedicine, vol. 15, pp. 163-172, 2017.

[70] C. P. Jenkinson, H. H. H. Göring, R. Arya, J. Blangero, R. Duggirala, and R. A. DeFronzo, "Transcriptomics in type 2 diabetes: bridging the gap between genotype and phenotype," Genomics Data, vol. 8, pp. 25-36, 2016.

[71] D. A. Winnier, M. Fourcaudot, L. Norton et al., "Transcriptomic identification of $\mathrm{ADH} 1 \mathrm{~B}$ as a novel candidate gene for obesity and insulin resistance in human adipose tissue in Mexican Americans from the Veterans Administration Genetic Epidemiology Study (VAGES)," PLoS One, vol. 10, no. 4, article e0119941, 2015.

[72] C. Bing, T. Mracek, D. Gao, and P. Trayhurn, "Zinc- $\alpha 2$-glycoprotein: an adipokine modulator of body fat mass?," International Journal of Obesity, vol. 34, no. 11, pp. 15591565, 2010.

[73] V. Ceperuelo-Mallafré, M. Ejarque, X. Duran et al., "Zinc- $\alpha 2$ Glycoprotein modulates AKT-dependent insulin signaling in human adipocytes by activation of the PP2A phosphatase," PLoS One, vol. 10, no. 6, article e0129644, 2015.

[74] S. Ge and A. S. Ryan, "Zinc- $\alpha 2$-glycoprotein expression in adipose tissue of obese postmenopausal women before and after weight loss and exercise + weight loss," Metabolism, vol. 63, no. 8, pp. 995-999, 2014.

[75] T. Gohda, Y. Makita, T. Shike et al., "Identification of epistatic interaction involved in obesity using the KK/Ta mouse as a type 2 diabetes model: is $\mathrm{Zn}-\alpha 2$ glycoprotein- 1 a candidate gene for obesity?," Diabetes, vol. 52, no. 8, pp. 2175-2181, 2003.

[76] K. S. Burgdorf, A. P. Gjesing, N. Grarup et al., "Association studies of novel obesity-related gene variants with quantitative metabolic phenotypes in a population-based sample of 6,039 Danish individuals," Diabetologia, vol. 55, no. 1, pp. 105113, 2012.

[77] M. C. Cornelis, E. B. Rimm, G. C. Curhan et al., "Obesity susceptibility loci and uncontrolled eating, emotional eating and cognitive restraint behaviors in men and women," Obesity, vol. 22, no. 5, pp. E135-E141, 2014.

[78] B. Namjou, M. Keddache, K. Marsolo et al., "EMR-linked GWAS study: investigation of variation landscape of loci for body mass index in children," Frontiers in Genetics, vol. 4, p. 268, 2013.

[79] Q. Hu, H. Chen, Y. Zuo et al., "Role of PCK1 gene on oil teainduced glucose homeostasis and type 2 diabetes: an animal experiment and a case-control study," Nutrition \& Metabolism, vol. 16, no. 1, p. 12, 2019.

[80] Y. Li, W. Ma, C. Xie et al., "Identification of genes and signaling pathways associated with diabetic neuropathy using a weighted correlation network analysis: a consort study," Medicine, vol. 95, no. 47, article e5443, 2016.

[81] Y. Huo, X. Guo, H. Li et al., "Targeted overexpression of inducible 6-phosphofructo-2-kinase in adipose tissue increases fat deposition but protects against diet-induced insulin resistance and inflammatory responses," The Journal of Biological Chemistry, vol. 287, no. 25, pp. 21492-21500, 2012. 
[82] T. Atsumi, T. Nishio, H. Niwa et al., "Expression of inducible 6phosphofructo-2-kinase/fructose-2,6-bisphosphatase/PFKFB3 isoforms in adipocytes and their potential role in glycolytic regulation," Diabetes, vol. 54, no. 12, pp. 3349-3357, 2005.

[83] X. Guo, K. Xu, J. Zhang et al., "Involvement of inducible 6phosphofructo-2-kinase in the anti-diabetic effect of peroxisome proliferator-activated receptor gamma activation in mice," The Journal of Biological Chemistry, vol. 285, no. 31, pp. 23711-23720, 2010.

[84] H. Dong, Y. Zhang, J. Wang et al., "Regulator of G protein signaling 2 is a key regulator of pancreatic $\beta$-cell mass and function," Cell Death \& Disease, vol. 8, no. 5, article e2821, 2017.

[85] H. Wang, J. H. Lee, and Y. Tian, "Critical genes in white adipose tissue based on gene expression profile following exercise," International Journal of Sports Medicine, vol. 40, no. 1, pp. 57-61, 2019.

[86] L. Gillberg, A. Perfilyev, C. Brøns et al., “Adipose tissue transcriptomics and epigenomics in low birthweight men and controls: role of high-fat overfeeding," Diabetologia, vol. 59, no. 4, pp. 799-812, 2016. 